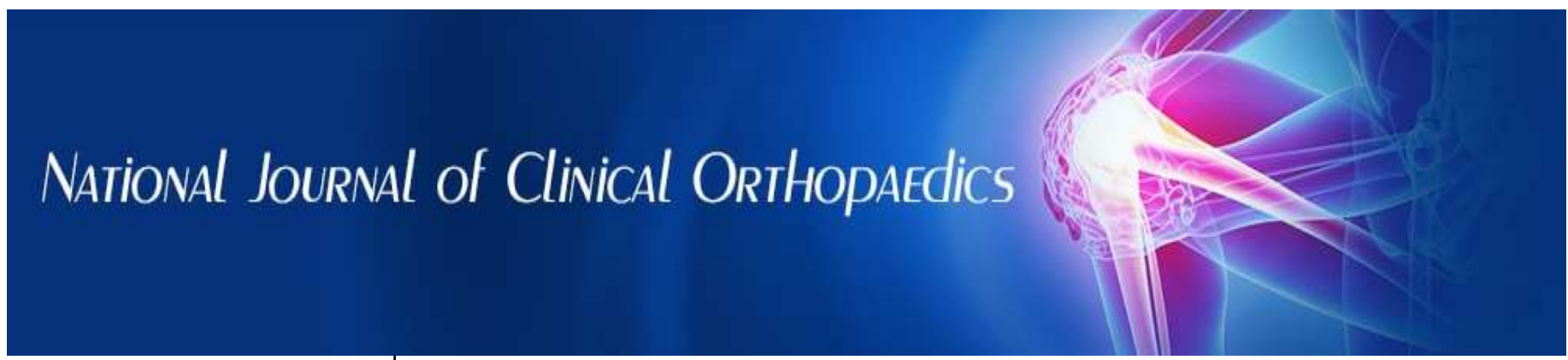

ISSN (P): 2521-3466

ISSN (E): 2521-3474

(C) Clinical Orthopaedics

www.orthoresearchjournal.com

2021; 5(4): 91-97

Received: 10-08-2021

Accepted: 13-09-2021

Dr. Vadhiraj Krishna JB Consultant Orthopedic Surgeon, Balaji Multispeciality Hospital, Hindupur, Andhra Pradesh,

India

Dr. Shreesh Kadur JM

Consultant Orthopedic Surgeon, Balaji Multispeciality Hospital,

Iritty, Kerala, India

Dr. Rohan Natthuji Kumre Senior Resident, Mallya

Hospital, Bengaluru,

Karnataka, India
Corresponding Author: Dr. Vadhiraj Krishna JB Consultant Orthopedic Surgeon, Balaji Multispeciality Hospital, Hindupur, Andhra Pradesh,

India

\section{Study and analysis of effect of calcium sulphate augmented with antibiotics in orthopaedic practice}

\author{
Dr. Vadhiraj Krishna JB, Dr. Shreesh Kadur JM and Dr. Rohan Natthuji \\ Kumre
}

DOI: https://doi.org/10.33545/orthor.2021.v5.i4b.332

\section{Abstract}

Background: Open fractures, osteomyelitis, peri-prosthetic infections whether impending or established are challenging situations in orthopaedic practice. Prevention and eradication of infections is a difficult task and incomplete treatment can lead to devastating complications. Treatment protocol involves wound debridement in cases of osteomyelitis and peri-prosthetic infections, primary internal fixation of the open fracture and application of calcium sulphate augmented with antibiotics, is a line of treatment in most of the cases.

Aim: To study and analyse the effect of calcium sulphate augmented with antibiotics in orthopaedic practice.

Materials and Methods: Patients were admitted in Mallya Hospital, Bangalore in between June 2018 to December 2019. Sample size of 30 was calculated based on the formula. Patients with diagnosis of osteomyelitis, peri-prosthetic infection and open fracture were included in the study. The patients were recruited to the study after due consent. Clinical history was taken and patients were examined, laboratory parameters (TC, ESR, CRP) were taken preoperatively and radiological examination done. Patients were followed up with laboratory parameters (TC, ESR, CRP), radiographs for resorption of calcium sulphate and bone healing at 6 weeks, 3 months and 6 months postoperatively.

Results: Minimum age of the patient was 15 years and maximum 83 years, 2 patients were lost to follow up, 21 males and 7 female patients. Cases were divided depending on the type. Postoperatively laboratory parameters with statistical significant $\mathrm{P}$ values in cases of osteomyelitis, peri-prosthetic infection, open fracture seen in TC, ESR and CRP are as follows, osteomyelitis $(0.016,0.011,<0.001)$, Periprosthetic infection $(<0.001,<0.001,<0.001)$ and in open fractures $(<0.001,0,003,<0.001)$. Calcium sulphate resorption was assessed along with bone healing. Average Calcium sulphate re-sorption period was 6 weeks and average bone healing time was 6 months in fracture and cases with the bone void formation. There was statistically significant difference between all the groups when compared pre and postoperatively.

Conclusion: We concluded that antibiotic impregnated Calcium sulphate plays an important role in tackling the infection when used locally at the site of infection; it helps in wound healing and bone healing.

Keywords: Calcium sulphate, orthopaedic practice, Open fractures, osteomyelitis, peri-prosthetic infections

\section{Introduction}

Orthopaedic trauma and infections are very common in Indian scenario. Open fractures and infections whether impending or established are challenging situations in orthopaedic practice. Acute or Chronic post traumatic and postoperative infections especially osteomyelitis, periprosthetic infections are conditions associated with increased morbidity to the patient ${ }^{[1-3]}$. In cases of severe bony infections, impending sepsis and mortality are the issues of concern in orthopaedic practice.

Due to bony defects, soft tissue damage, dead space formation, severe contamination, poor vascularity, lowering the rate of infection is the main challenge. The current concept in the treatment of orthopaedic infections and open fractures include immediate aggressive debridement to remove suspicious things which can act as a potential risk for the infections. Early stabilization of the fracture, stabilization of the defect formed in cases in orthopaedic infections, osteomyelitis ${ }^{[4,5]}$. 
Stabilization of the open fracture or bony defect formed and bone loss in infections eg: osteomyelitis is essential for decreasing the pain, to decrease the chance of neurovascular injuries, soft tissue damage, wound healing and bone healing. It is difficult to eradicate/treat the osteomyelitis infection due to complexity of diagnosis, the nature of the condition, extent of the spread and line of management ${ }^{[6]}$.

Recently treatment of open fracture primarily with internal fixation has shown encouraging results ${ }^{[7]}$ but still there is controversy that internal fixation may increase the chance of infections due to bacterial adherence and biofilm formation on surface of metallic implants ${ }^{[8]}$. In peri-prosthetic infections there are difficulties in eradication of the infective focus, which is a concern whether to retain the implant or not.

The current treatment of open fracture along with post traumatic or post-operative osteomyelitis /infections, contaminated wound requires thorough surgical debridement, elimination of dead space formed by bone loss \& microbial infection, systemic antibiotics for longer duration in combination with local release of antibiotic obtained by implantation of the bio-absorbable materials like Calcium sulphate, bone graft substitutes ${ }^{[9-16]}$ and non-absorbable materials like Polymethylmethacrylate (PMMA) cement impregnated with antibiotics ${ }^{[17-20]}$.

Although the main line of treatment of open fracture and local infections is systemic antibiotic therapy but local antibiotic delivery has shown good results to tackle these situations very well ${ }^{[21-27]}$. In open fractures with bone loss, use of calcium sulphate with antibiotic impregnated PMMA is also used for primary stabilizations and to fill the defect ${ }^{[29,30]}$. Calcium sulphate has several advantages over other local antibiotic delivery methods.

It helps in new bone formation (osteoconduction) along with this, it also helps to fill the dead space formed due to bone loss occurred with trauma and surgical bone loss or infective conditions. With the increased number of complex trauma cases and chronic orthopaedic infections, patients have to undergo long term treatment (which may or may not benefit the patient). In long term treatment patients have to face other co-morbidities which hamper their moral and overall outcome also.

In cases of established orthopaedic infections, osteomyelitis, complete eradication of the infection avoiding subsequent need for surgery so as to decrease the morbidity and provide a cost effective treatment plan without increasing the burden on the patient.

\section{Materials and Methods}

Patients were admitted in Mallya Hospital, Bangalore in between June 2018 to December 2019. Sample size of 30 was calculated based on the formula. Patients with diagnosis of osteomyelitis, peri-prosthetic infection and open fracture were included in the study. The patients were recruited to the study after due consent. Clinical history was taken and patients were examined, laboratory parameters (TC, ESR, CRP) were taken preoperatively, radiological examination was done. Patients were assessed with laboratory parameters (TC, ESR, CRP), radiographs in anteroposterior and lateral views for resorption of calcium sulphate and bone healing, which formed the base line of study patients were followed up-to 6 weeks, 3 months and 6 months postoperatively.

\section{Surgical Procedure}

Depending upon the diagnosis of the conditions like

1. For open fracture with bone loss with or without established infection.

2. Established infections after primary fixation of the open fracture or closed fracture (periprosthetic postoperative infections)

3. Acute or chronic osteomyelitis.

Thorough preoperative evaluation/investigations were done in terms of hematological, microbiological and radiological examinations.

The aim of the treatment protocol was to find the usefulness of local use of antibiotic (vancomycin) impregnated calcium sulphate beads to prevent, eradicate infection and to aid wound healing process, to help [in healing of bony defect and fracture union with the help of systemic or oral antibiotics in combination in the treatment of open contaminated fractures, established postoperative (periprosthetic) acute infections, acute or chronic osteomyelitis].
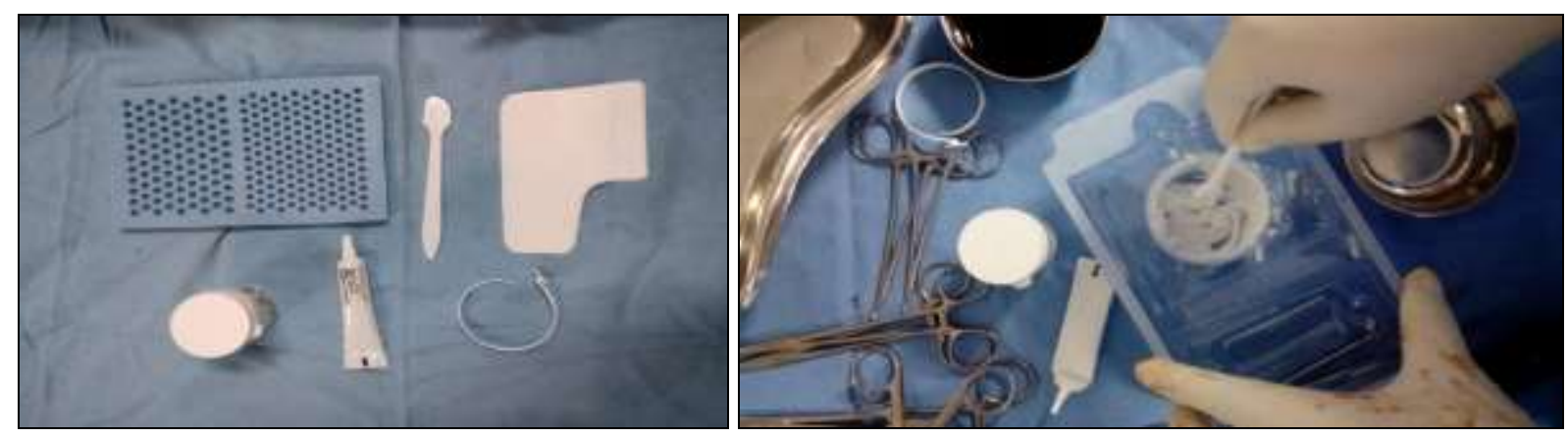

Calcium Sulphate Beads Preparation Kit

The Treatment Protocol: We used Synthetic calcium sulphate available in market- 5 to 10 grams of calcium sulphate in addition to 1 gram of vancomycin powder mixed with the solvent and moulded into small pellets which were plaCED at the site of the infection, bone void formed at the fracture site and also in cases of osteomyelitis.

1. Surgical debridement of the wound, includes resection of the soft tissues, thorough curettage, full wash, prevention of vascularity in open fracture, filling the defect formed due to bone loss with calcium sulphate with or without PMMA antibiotic loaded cement and internal fixation with plate and screws.

2. In few cases of open wound with severe bone loss, primary stabilization of the fracture with external fixator and thorough surgical debridement, filling the defect formed with calcium sulphate augmented with $1 \mathrm{gm}$ of vancomycin, covering the soft tissue defect with vascularized muscular flaps or split skin graft to aid in healing.

3. In cases with acute periprosthetic infections formed immediately after primary fixation, treatment with 
debridement, retention or removal of implant depending on the radiological signs, excision of the infection tract, removal of potential causes for local infections in combination of systemic antibiotics for longer duration.

4. In cases of acute or chronic osteomyelitis, drainage of pus, debridement, curettage of the infected bone or soft tissue filling the defect formed with antibiotic loaded calcium sulphate beads and culture sensitive systemic antibiotics for longer duration.

\section{Post - Operative Protocol}

Postoperatively hematological investigations such as CBC, ESR, CRP at 3 weeks, 6 weeks, 3 months and 6 months. Patient were again clinically evaluated to check the surgical wound and signs

of infection. Local examination of the operated site to notice any discharging pus or fluid. Postoperatively regular wound inspection and dressings were done, parenteral antibiotics were given initially and then oral antibiotics as per the culture and sensitivity of the antibiotic to the particular organism with antiinflammatory analgesics for more symptomatic relief.

Postoperative radiographs were taken to rule out any infection, resorption of calcium sulphate and radiological signs of bone healing reconstruction and stability at the operated site. Then mobilization started with the definitive fixation methods for fractures and supportive measures for other infections.

\section{Results}

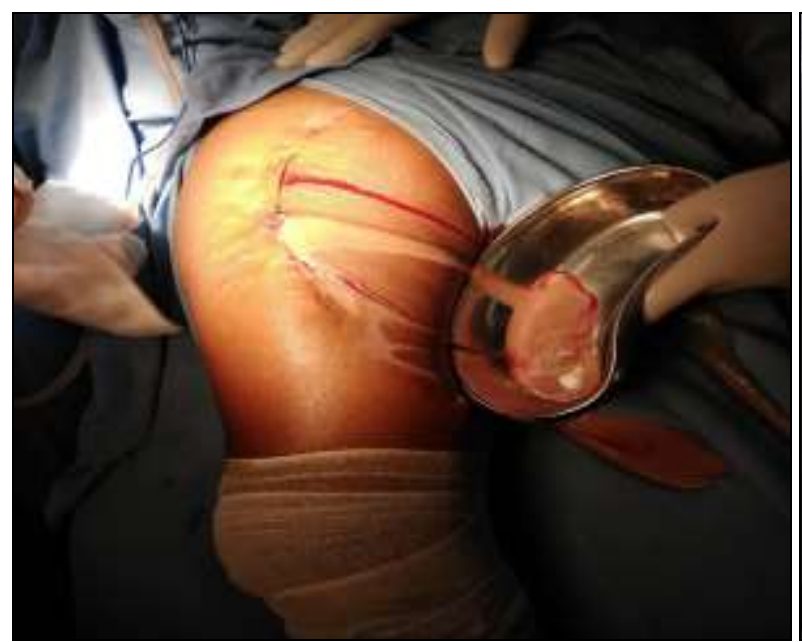

Pus draining out

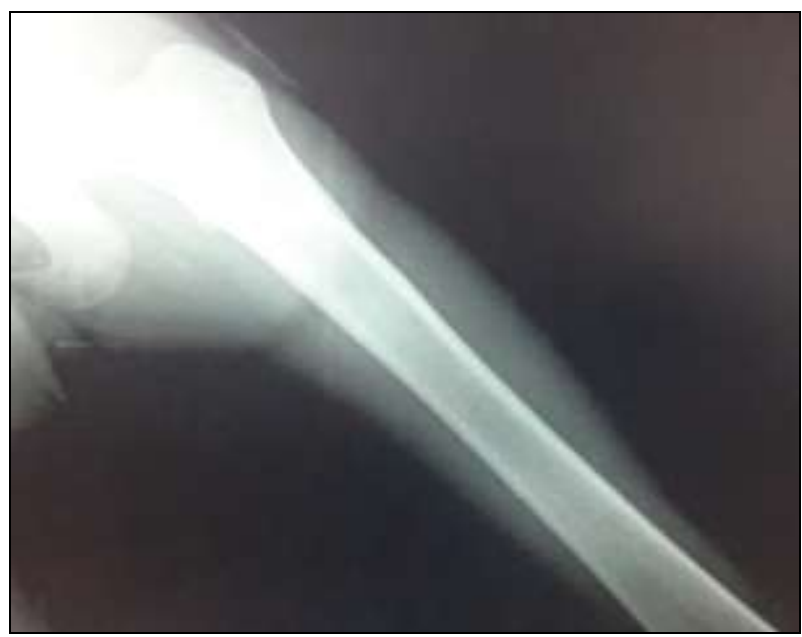

6 months follow up

28 cases were included in our study, all were classified into various types depending on the condition, there were 6 cases of osteomyelitis, 11 cases of periprosthetic fracture, 10 cases of open fracture and 1 case of de-gloving injury to the left leg. All the cases were evaluated preoperatively, underwent surgical procedure with application of $1 \mathrm{gm}$ of vancomycin impregnated calcium sulphate pellets. All the cases were followed up for 6 months except 1 case, who expired in the course of time due to medical co-morbidities. We achieved good result in 25 cases. 2 cases went into failure.

In 10 cases of open fractures we achieved $100 \%$ control in infection rate, in 8 cases we were able to notice good bone healing, 2 cases went for further reconstructive surgeries. In 1

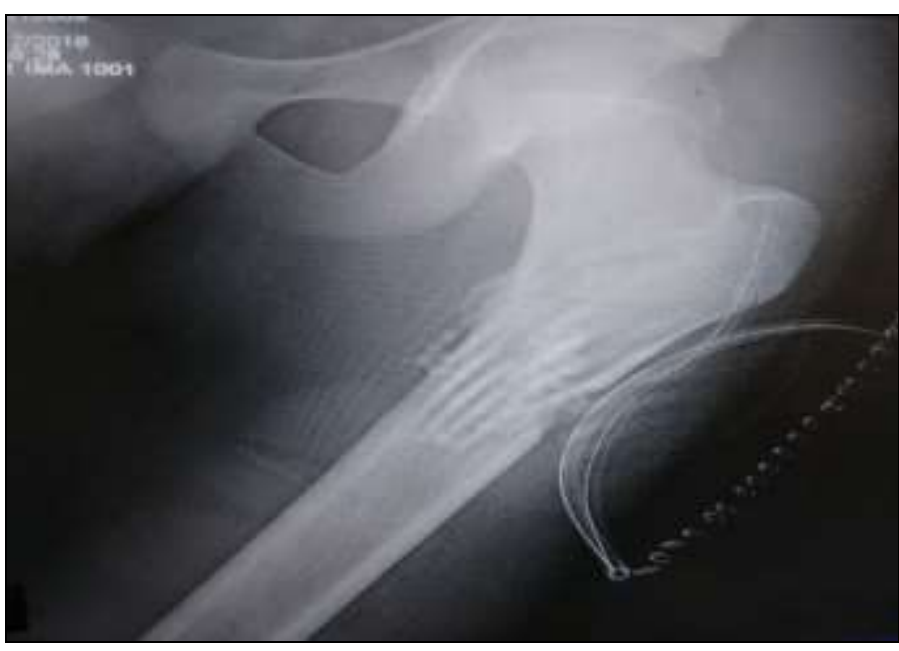

calcium sulfate $+1 \mathrm{~g}$ vancomycin applied

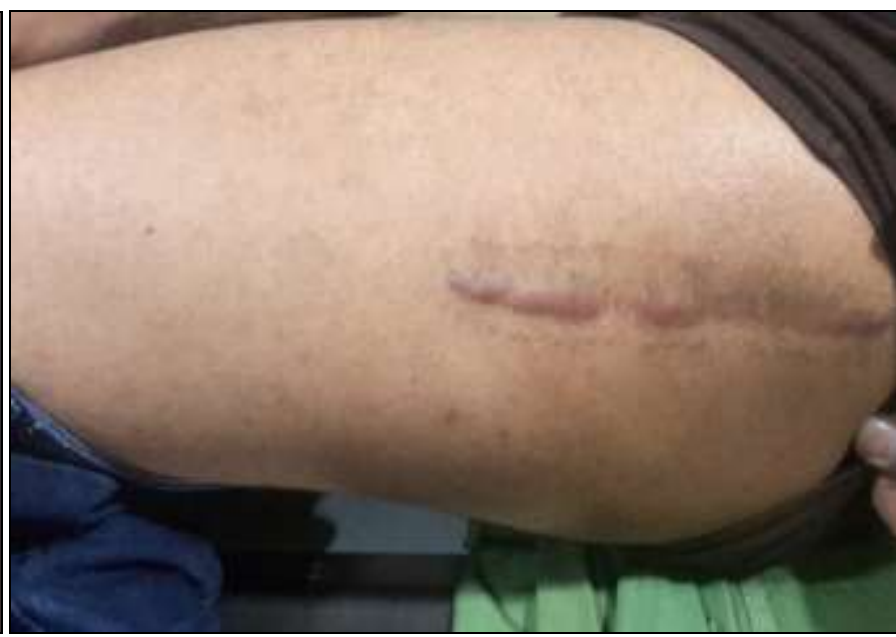

healed wound at 6 weeks followup

case of open fracture we used antibiotic loaded PMMA cement spacer to fill the bony defect formed at the time of trauma due to bone loss, along with calcium sulphate augmenting the internal fixation and primary closure at the primary stage, in $2^{\text {nd }}$ stage we removed the cement spacer. And other case of compound fracture distal end of femur and proximal tibia which was fixed with external fixator, muscle pedicle graft and skin graft was reoperated after total control of infection for realignment of the fracture fragment in further stage.

In 11 cases of peri-prosthetic infection 9 cases were successfully recovered within the time period of 6 months in addition to the antibiotics given for longer duration. Wounds were completely healed with further signs of inflammation. 1 case of peri- 
prosthetic infection of postoperative Total Knee Arthroplasty went into failure due to resistant organism to antibiotics, which further require surgery implant removal+ antibiotic loaded PMMA cement spacer application and covering the soft tissue defect formed over the knee with rotational musculocutaneous flap. 1 case of periprosthetic infection has implant loosening due to accidental weight bearing few days after postoperatively, which was treated with cylinder cast for additional stability.

1 case went into failure and expired in the course due to medical co-morbidities and septicemia developed in the patient.

6 cases of osteomyelitis were followed, 1 case of tubercular osteomyelitis showed complete recovery after 6 months of AKT in adjunct to surgical debridement and vancomycin impregnated calcium sulphate application. 4 others cases of osteomyelitis showed good recovery till the time of their follow up. 1 case of osteomyelitis of calcaneum went into failure, due to uncontrolled diabetes and peripheral vascular disease which required amputation.

laboratory parameters with statistical significant $\mathrm{P}$ values in cases of osteomyelitis, peri-prosthetic infection, open fracture was seen in TC, ESR and CRP are as follow, osteomyelitis $(0.016, \quad 0.011,<0.001)$ Peri-prosthetic infection $(<0.001$, $<0.001,<0.001)$ and in open fractures $(<0.001,0,003,<0.001)$ were noted.

Over all in most of the cases wound healing was good with no signs of inflammation and infection. Most of the cases of open fractures and postoperative peri-prosthetic infection showed good bone healing in average time period of 6 months (range 49 months). Re-sorption of calcium sulphate pellets was evident in average 6 weeks (range 3 weeks- 3 months). 3 cases had serous drainage following the implantation of the calcium sulphate pellets, in those cases wound healing was observed in 3 weeks.

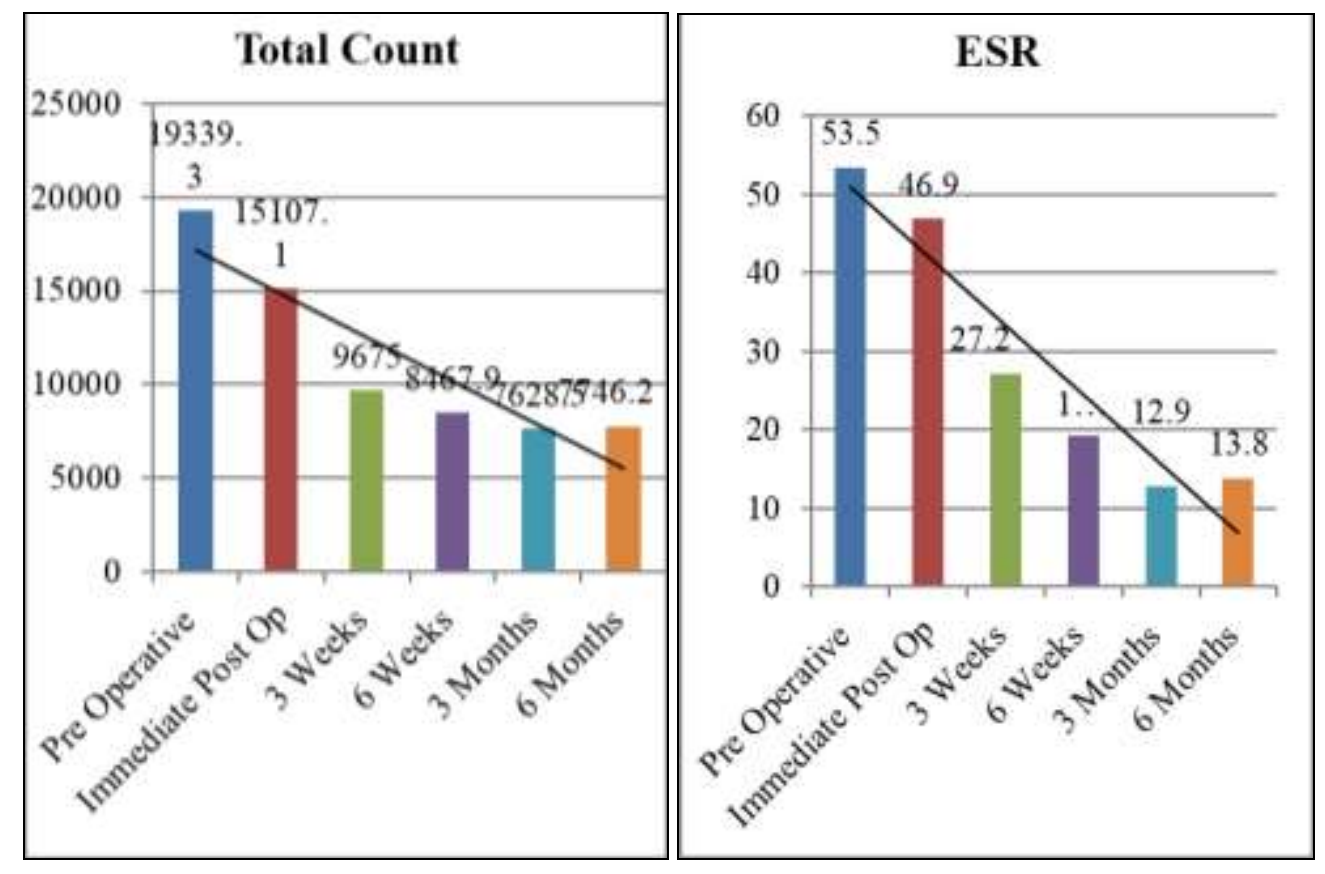

Bar Graph Depicting the Total Count and Esr Count
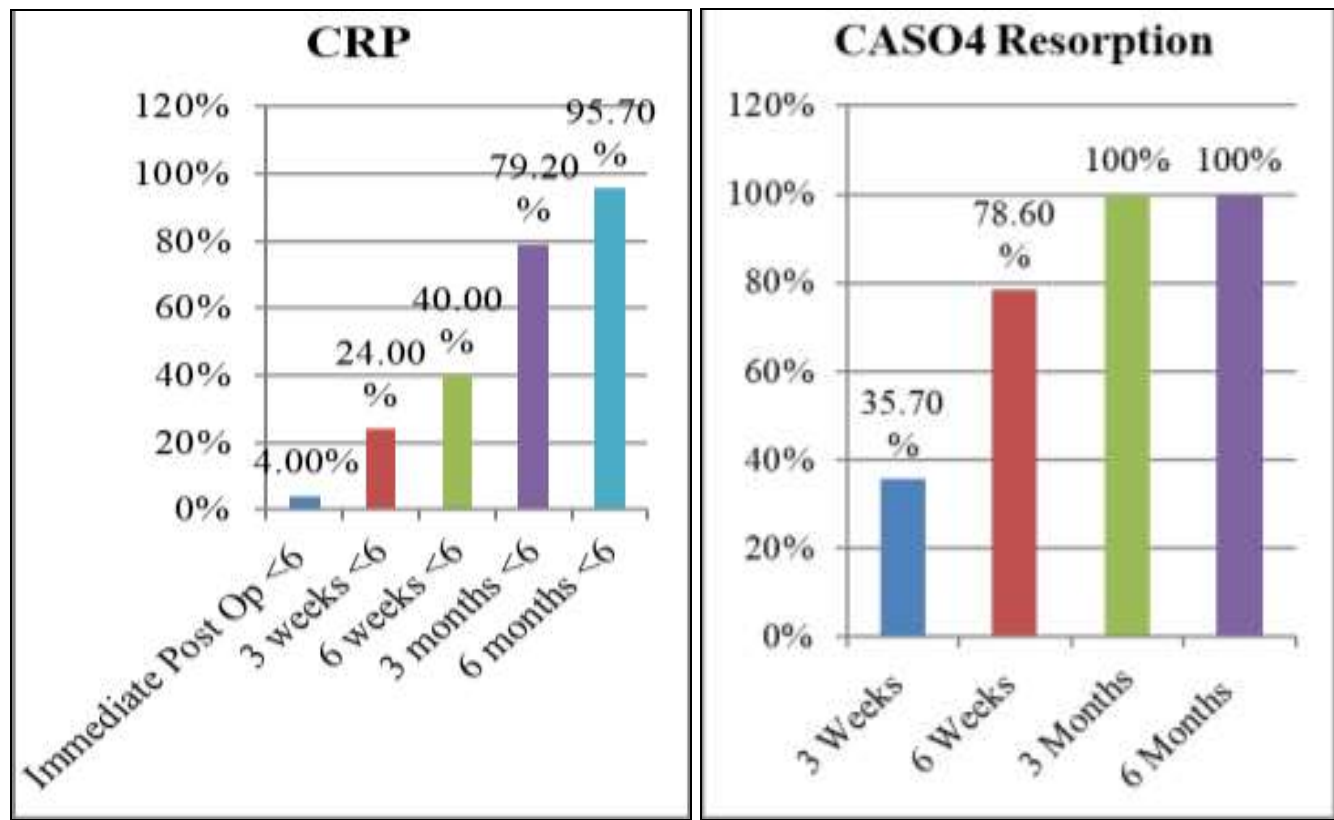

Bar Graph Depicting the CRP Count and Calcium Sulphate Resorption Time 


\section{Discussion}

Treatment of open fractures is challenging and, in few cases, it is difficult to salvage the limb also. The mode of fracture fixation has changed to internal fixation from external fixation which is quite evident in recent years, which as shown great results. However, infection is the most common thing associated with open fractures followed by nonunion and mal-union. These things should be tackled in such a way to reduce the risk at the time of primary surgery itself. The importance of antibiotics is very much evident in orthopaedic practice, initial doses of intravenous antibiotics play important role in controlling the infection. Antibiotics alone have been associated in decreasing the incidence of infection from 13.9 to $2.3 \%$ but high doses of systemic antibiotics and longer duration of treatment required to get sufficient local antibiotic level at the site of infestation for eradication of the infection. Local delivery system of antibiotics with the use of Calcium sulphate and other bone graft substitutes as a biodegradable and biocompatible material has shown great results in few of the studies done in the past. Use of Calcium sulphate could avoid the potential complication of infection and helps in new bone formation due to its osteo-conductive properties.

Cai X et al. ${ }^{[23]}$ studied on 28 patients of open fracture of long bone, which were treated with internal fixation evaluated bone healing and absorption of calcium sulphate. 26 of those patients followed successfully. There was no evidence of incidence in 26 cases. Exudation was observed in 2 cases. Average resorption time of calcium sulphate was 1.4 months and average bone healing healing was 5.8 months (range 4-9 months). The study shows that combination of internal fixation with vancomycin impregnated Calcium sulphate decreases the incidence of deep infection. Helgeson et al. ${ }^{[7]}$ reported in their study, where they treated 17 combat related open fractures along with antibiotic impregnated calcium sulphate in different bones, in 12 fractures with average 8.5(1-19 months) months of follow up demonstrated clinical and radiological evidence of fracture healing and consolidation.5 of the patients required amputation, 3 for persistent infection, 2 for severe neurogenic pain.

Zalvarus CG et al. ${ }^{[24]}$ used local antibiotics in open fractures and osteomyelitis with bioabsorbable and nonabsorbable material, found local antibiotic therapy is safe technique, has high local concentration with minimal systemic levels and eliminate the need for reoperation and removal. Kelly CM et al. ${ }^{[36]}$, in their study on 109 patients with bone defects occurred due to trauma, peri-prosthetic bone loss, tumor or fusion. Only Calcium sulphate alone was used. In their follow up of 12 months, they noted $99 \%$ of the calcium sulphate was absorbed in 6 months, $88 \%$ of the defect was filled with trabeculated bone. 4 cases shown complications.

In our study of open fractures where 10 cases were studied, which were treated with internal fixation and antibiotic loaded calcium sulphate application, all cases had complete calcium resorption by 3 months with average resorption time of 6 weeks, 8 of the cases of open fracture showed complete union by average 6 months (range 5-9 months), where 2 cases were reoperated for corrective surgeries, 1 case with realignment of the facture fragment. Other case was operated with vascularized fibular graft for bony defect in femur. All of the treated fracture cases showed complete wound healing. Thus our study simulated the above mentioned studies that antibiotic loaded Calcium sulphate helps in infection control and new bone reconstruction in open fracture cases when treated well.

Peri-prosthetic joint infections are challenging in orthopaedic practice, eradication of complete infection is difficult, also requires long term follow up. It can be a potential threat to the limb or life of the patient. Edward J Mc Pherson et al. ${ }^{[35]}$ in their study population of 250 patient where they studied on use of dissolvable antibiotic beads in treatment of periprosthetic joint infections and revision arthroplasty, used vancomycin and tobramycin, they found successful results in most of the cases, failure percentage in those cases where drainage from the surgical wound $3.2 \%$, which was evident with use of higher volume of bead use. Heterotropic bone formation was seen in $1.2 \%$ cases. Out of 250 , only 9 has failure due to infection. They found antibiotic loaded synthetic calcium sulphate beads are acceptable delivery tool for local antibiotic in cases of revision joint cases.

Fleiter $\mathrm{N}$ et al. [33] in their study in 20 posttraumatic/ postoperative bone infection where they used antibiotic loaded calcium sulphate after radicle debridement assessed wound healing, infection parameters (lab parameters), resorption of calcium sulphate beads and bone union found sufficient active local levels of antibiotic and simultaneous low systemic exposure showed that material used was safe and showed no recurrence of infection.

Toms AD et al. ${ }^{[22]}$, in 3 years of their study on 140 total hip arthroplasty patient, found infection rate in $7-16 \%$ who underwent revision found that increased level of ESR(erythrocyte sedimentation rate/hour) $>30$ and $\mathrm{CRP}(\mathrm{C}$ reactive protein.) $>10 \mathrm{mg} / \mathrm{l}$, have probability of infection rate in $83 \%$ cases, and when both are negative infection may be reliably excluded.

In our study of 11 cases of peri-prosthetic infections 9 cases were treated successfully which was evident with the lab parameters, TC, ESR, CRP, and clinical signs of infection for the period of 6 months, which is comparative with most of the above studies. 2 cases went into failure, 1 patient required 2 stage $2^{\text {nd }}$ revision surgery and 1 patient lost in the follow up due to death.

Osteomyelitis remains as a challenge despite developmental advances in antibiotics and surgical techniques good cure can be achieved with antibiotic therapy in most of the cases of acute osteomyelitis but should be diagnosed early. The treatment of chronic osteomyelitis is surgical with antibiotic therapy in addition. Surgical debridement can give good results when treated in combination with local antibiotic when used with bioabsorbable and non-absorbable material augmented with antibiotics. Bone graft substitutes can also aid in achieving good results.

Mc Nally et al. ${ }^{[34]}$, studied on 100 cases of osteomyelitis, 81 cases following injury and surgery, cases were followed average 19.5 months (range 12-34 months). In 96 patients infection was eradicated with single stage procedures, 4 cases had recurrence which were successfully managed in $2^{\text {nd }}$ surgery, adverse effects were noticed as fractures in 3 cases, 6 wound leaks and 3 unrelated deaths, concluded that single stage surgical protocol along with absorbable local antibiotics is effective and offers patient friendly treatment.

Yan RJ et al. ${ }^{[28]}$, in single stage compound grafting of antibiotic impregnated calcium sulphate and autologous cancellous bone for the treatment of chronic osteomyelitis in 52 cases in period of 2 years and followed them for average 2.8 years (2-3.8yrs) noticed primary healing was achieved in 52 cases, 2 cases went into recurrence. Bone repair was gained in 1.5-3.5 months (avg 2.5 months), complete radiological absorption of calcium sulphate in 1.2 to 3 months (avg 2.2 months), persistence of drain was in 10 cases for 2 to 3 months. Mean Maryland score was $88.15+/-7.70$. There were excellent results in 32 case, good 
in 14 and fair in 6 cases.

Ferguson et al. ${ }^{[32]}$, in their study population of 193 patient of osteomyelitis, who underwent surgical debridement and bioabsorbable calcium sulphate antibiotic carrier, where they reviewed to determine rate of infection, filling of bone defects and wound healing. In the different study groups they found that infection was settled in almost all cases, prolonged oozing noted in 30 cases, there was no evidence of recurrent infection. Defect formed after debridement with no healing in 67(36.6\%), partial healing in $108(59 \%)$ and complete healing (4.4\%) which shows variability in bone defect filling.

In our study, 6 cases of osteomyelitis was included, 5 cases did show good results in terms of wound healing, average calcium sulphate re-sorption was 6 weeks ( 3 weeks to 3 months), new bone formation was evident in average 6 months(5-9 months). 1 patient underwent amputation due to uncontrolled diabetes and peripheral vascular disease comparable with above mentioned studies.

\section{Conclusion}

In the study we conducted, we found that Antibiotic impregnated Calcium sulphate plays an important role in tackling the infection when used locally at the site of infection, it helps in bone healing. The short term results are favourable but requires long term follow up to evaluate the effectiveness of the locally used Calcium sulphate in eradication of infection in long run.

\section{Recommendations}

We recommend the use of calcium sulphate impregnated with antibiotic for local delivery of the drug in cases such as periprosthetic infections, osteomyelitis and open.

\section{References}

1. Cierney G, Mader JT, Penninck JJ. a clinicalstaging system foe adult osteomyelitis. Clinorthop res 2003;(414):7-24.

2. Mcpherson EJ, Peters CL, Musculoskeletal infection. In: Flynn JM. ed Orthopaedic knowledge update. $10^{\text {th }}$ ed. Rosemont, IL: American academy of Orthopaedic surgeon, 2011, 239-58.

3. Kurtz SM, Ong KL, Lau E. Prosthetic joint infection risk after TKA in the medicare population. Clinical orthopaedic and related research 2010;468:52-56.

4. Trampuz A, Zimmerli W. diagnosis and treatment of implant associated septic arthritis and osteomyelitis. Curr infect dis Rep 2008;382:394-403.

5. Edwards J, Keni O, Martini M, Conort O. Treatment of chronic osteomyelitis by antibiotic loaded plaster of Paris pellets. Patho. biol 1990;38:5543-7.

6. Frosberg JA, Potter BK, Cierny G, Webb L. diagnosis and management of chronic infection. J AM Acad orthop surg 2011;19(1):S8-19.

7. Helgeson M, Potter B, Tucker C, Frisch HM. Antibiotic impregnated calcium sulphate use in combat related open fracture. Orthopedics 2009;32:323.

8. Costerton JW, Stewart PS, Greenberg EP. Bacterial biofilm: common cause of persistent infection. Science 1999;284:1318-22.

9. Malle GE. The use of antibiotic loaded synthesized calcium sulphate pellets in theone stage treatment of osteomyelitis, paper presented at:annual open scientific meeting of musculoskeletal infection society, proceeding of the $19^{\text {th }}$ meeting of skeletal infection society august 7-8. San Diego California 2009.
10. Mousset B, Benoit MA, Delloy C, Banillet R, Gillard J. Biodegradable implants for potential use in bone infection. An invitro study of antibiotic loaded calcium sulphate. International orthopaedic 1995;19:157-61.

11. Thomas MV, Puleo DA, Al- Sabbagh M. calcium sulphate:a review. J long termeffect med implants 2005;15(6):5999607.

12. Thomas DB, Brooks DE, Wenke JC, Bice TG, lonergan KT. Tobramycin impregnated calcium sulphate prevents infections in contaminated wounds. Clin orthop relat res 2005;441:366-71.

13. Sulo L, use f gentamicin impregnated plaster of paris beads in treatment of bone infection. Revue de cherugie orthopedique 1993,79,299-305.

14. Papagelopoulous PJ, Mavrogenis AF, Tsiodras et al. calcium sulphate delivery system with tobramycin for treatement of chronic psteomyelitis. The journal of international. Medical research 2006;34:704-712.

15. Pietrzak WS, Ronk R. Calcium sulphate bone void filler:a review and look ahead, journal of craniofacial surgery 2000;11(4):327-333.

16. Lalidau F. bone infection and bone graft substitutes for local antibiotic therapy.

17. Bowyer GM, Cumberland N. Antibiotic release from the impregnated pellets and beads. The journal of trauma 1994;36:3331-5.

18. Raushmann MA, Wichelhaus TA, Stirnal V, Dingeldein E, Zichner L, Schetner RA. Nanocrystalline hydroxyapatite and calcium silphate as biodegradable composite carrier material for local delivery of antibiotics in bone infections. Biomaterials 2005;26(15):2677-84.

19. Chang W, Colangeli M, Colangeli S, Dibella C, Gozzi. Donati D. Adult osteomyelitis; debridement vs debridement plusosteoset T pellets. Acta orthop Belg 2007;73:238-43.

20. Canavese F. Successful treatment of chronic osteomyelitis in children with debridement, antibiotic laden cement spacer and bone graft substitute. Eur. J Ortho. Surg. Traumatol 2017.

21. Dacquet V, Varlet A, Tandogan $\mathrm{RN}$ et al- antibiotic impregnated plaster of paris beads. Trails with Teicoplanin. Clinical orthop rel res 1992;282:241-9.

22. Toms AD, Davidson D, Masri MA, The management of periprosthetic infection in total joint arthroplasty. The journal of bone and joint surgery. British. 2006:88(2):149155.

23. Cai X, Han K, Cing K, Cai J, tong D, Han D et al. The use of calcium sulphate impregnated with vancomycin in the treatement of open fracture of long bone: a preliminary study. Orthopedics 2010;33(3). doi

24. Zalvarus CG, Patzakis MJ, Webb JJ, Holtom P. local antibiotic therapy in treatement of open fractures and osteomyelitis. Clin orthop relat res 2004;427:86-93.

25. Turner TM, Urban RM, Hall DJ, Chye PC, Segreti J, Gitelis S. local systemic levels of tobramycin delivery from calcium sulphate bone graft substitute pellets. Clin ortho relat res 2005;437:97-104

26. Bibbo C. DV Patel- the effect of demineralized bone matrix - calcium sulphate with vancomycin on calcaneal fracture healing and infection rates:prospective study.

27. Drosos GE, Baboura EC, Ververidid A, Kalkagia D, Veretas DA. Calcium sulphate cement in contained traumatic metaphyseal bone defects. Surg technol. Int. 2012;22:313-319.

28. Yan RJ. one stage compound grafting of antibiotics 
impregnated calcium sulphate and autologous cancellous bone for the treatement of chronic calcaneal osteomyelitisZhoungguo GU, Shang.

29. Evans RP, Nelson CL. Gentamycin - impregnated PMMAbead compared with systemic therapy in the treatment of chronic osteomyelitis. Clinical orthopaedic related research 1993, 295.

30. Henry SI, Hood G, Seligson D. long term implantation of gentamicin PMMA beads- clinical orthopedics and rela res 1993;295:47-53.

31. McLaren AC, Alternative materials to acrylic bone cement for delivery of depot antibiotics in orthopedic infections. Clin orthop relat res 2004;427:101-106.

32. Ferguson JY, Dudareva M, Riley ND, Stubbs D, Atkins BL, McNally MA. The use of a biodegradable antibiotic-loaded calcium sulphate carrier containing tobramycin for the treatment of chronic osteomyelitis: a series of 195 cases. Bone Jt J. 2014;96-B(6):829-36.

33. Fleiter N, Walter $\mathrm{G}$, Bösebeck $\mathrm{H}$, Vogt S, Büchner H, Hirschberger W, et al. Clinical use and safety of a novel gentamicin-releasing resorbable bone graft substitute in the treatment of osteomyelitis/osteitis. Bone Jt Res 2014;3(7):223-9.

34. McNally M, Small J, Tofighi H, Mollan R. Two-stage management of chronic osteomyelitis of the long bones. The Belfast technique. J Bone Joint Surg Br. 1993;75$\mathrm{B}(3): 375-80$.

35. Edward McPherson MF, Matthew Dipane BA, Sherif Sherif MD. Dissolvable Antibiotic Beads in Treatment of Periprosthetic Joint Infection and Revision Arthroplasty The Use of Synthetic Pure Calcium Sulfate (Stimulan®) Impregnated with Vancomycin \& Tobramycin. Reconstr Rev [Internet] 2013, 19. [cited 2019 Jul 21];3(1).

36. Kelly CM, Wilkins RM, Gitelis S, Hartjen C, Watson JT, Kim PT. The Use of a Surgical Grade Calcium Sulfate as a Bone Graft Substitute: Results of a Multicenter Trial. Clin Orthop Relat Res 2001;382:42. 